\title{
Origin of rabbit (Oryctolagus cuniculus) in China: evidence from mitochondrial DNA control region sequence analysis
}

\author{
J.-R. Long ${ }^{*,+}{ }^{\ddagger}$, X.-P. Qiu ${ }^{\ddagger}$, F.-T. Zeng ${ }^{\ddagger}$, L.-M. Tang ${ }^{\dagger}$ and Y.-P. Zhang* ${ }^{*, \S}$ \\ *Yunnan Laboratory of Molecular Biology of Domestic Animals, Kunming Institute of Zoology, Chinese Academy of Sciences, Kunming, \\ 650223, China. ${ }^{\dagger}$ Sichuan Academy of Animal Sciences, Chengdu, Sichuan, 610066, China. ${ }^{\ddagger}$ Sichuan Agricultural University, Ya'an, Sichuan, \\ 625014, China. ${ }^{\S}$ Laboratory for Conservation and Utilization of Bio-resource, Yunnan University, Kunming, 650091, China
}

\section{Summary}

\begin{abstract}
A fragment of mitochondrial DNA (mtDNA) control region $(\sim 700 \mathrm{bp})$ was sequenced in 104 individuals from 20 breeds (three Chinese domestic breeds, five recently derived breeds and 12 introduced breeds) of domestic rabbits, Oryctolagus cuniculus. Nineteen sites were polymorphic, with 18 transitions and one insertion/deletion, and eight haplotypes (A1, A2, A3, A4, A5, A6, A7 and A8) were identified. Haplotype A1 was the most common and occurred in 89 individuals. In the 25 Chinese rabbits, only haplotype A1 was observed, while four haplotypes (A1, A3, A5 and A6) were found in 26 recently derived individuals. Haplotype A2 was shared by seven individuals among three introduced strains. The other six haplotypes accounted for $0.96-1.92 \%$ of the animals. Combined with the published sequences of European rabbits, a reduced median-joining network was constructed. The Chinese rabbit mtDNAs were scattered into two clusters of European rabbits. These results suggest that the (so-called) Chinese rabbits were introduced from Europe. Genetic diversity in Chinese rabbits was very low.
\end{abstract}

Keywords control region, genetic diversity, mtDNA, origin, rabbit.

\section{Introduction}

Knowledge of origin and evolution of domesticated species is not only an important academic question, but also has practical value for informed conservation of genetic diversity. Chinese rabbits have some desirable production traits, such as high reproductivity and disease resistance (Ban et al. 1996). However, the origin and genetic differentiation among the Chinese breeds is debated (Luo 1988; Chen \& Wang 1991; Chen 1984). Some believe Chinese rabbits were introduced from Europe, perhaps through the Silk Road, because no fossils of domesticated or wild rabbits (Oryctolagus cuniculus) have been found in China so far (Luo 1988). Another hypothesis is that Chinese rabbit originated in China (Chen 1984; Chen \& Wang 1991). Chen (1984 and references in) suggested that the lack of rabbit fossils

Address for correspondence

Ya-ping Zhang, Yunnan Laboratory of Molecular biology and Domestic Animals, Kunming Institute of Zoology, Chinese Academy of Sciences, Kunming, 650223, China.

E-mail: zhangyp@public.km.yn.cn

Accepted for publication 8 September 2002 was because the bones of rabbits were small, brittle and difficult to retain in fossils. This may be true for some of the bones, but not all, as rabbit remains were extremely abundant at many archaeological sites dating back over 10000 years and even earlier in Europe (Hardy et al. 1995). However, both hypotheses were based on limited genetic evidence.

Analysis of mitochondrial DNA (mtDNA) is useful for investigating the genetic variations and evolutionary relationships of animals because of its specific characteristics such as high variability and maternal transmission. It has been widely used in the genus Oryctolagus cuniculus and suggests that modern European rabbits had the same roots as the European wild rabbits, O. cuniculus, in the Iberian Peninsula (Ennafaa et al. 1987; Mignotte et al. 1990; Hardy et al. 1995; Monnerot et al. 1994, 1996; Fuller et al. 1997).

At the present time, there are about 20 rabbit breeds (strains) in China. Some of them were European breeds imported into China, while other breeds are believed to be Chinese breeds or strains. Recently derived breeds have been developed in recent years using crossbreeding and genetic selection. In this study, we sequenced about $700 \mathrm{bp}$ of mtDNA control region of 104 individuals from 20 breeds or 
strains collected in China, with the objective of elucidating the origin of Chinese rabbits.

\section{Materials and methods}

\section{Sampling}

Whole blood, white cells and muscle samples of 104 individuals from 20 breeds (three Chinese breeds, five recently derived breeds and 12 introduced breeds) were collected from seven provinces of China. The locations and the sample sizes of each breed or strain are listed in Table 1.

\section{DNA amplification and sequencing}

Total DNA was extracted by standard phenol/chloroform methods. A 700-bp fragment of mtDNA control region near the tRNA-Pro gene was amplified using primers L15438 (5'-GCTGATATTCTACTTAAACTA-3') and H16138 (5'-AGGGTCTTCATTAGGTGCTCGTCT-3'). Nucleotide positions were numbered according to the complete rabbit mtDNA sequence (Gissi et al. 1998; GenBank accession number NC-001913). Polymerase chain reaction was performed using about $20 \mathrm{ng}$ of DNA in a $25-\mu \mathrm{l}$ reaction volume with 35 cycles $\left(94{ }^{\circ} \mathrm{C}\right.$ for $1 \mathrm{~min}, 50{ }^{\circ} \mathrm{C}$ for $1 \mathrm{~min}$ and $72{ }^{\circ} \mathrm{C}$ for $1 \mathrm{~min})$. The products were purified with a gel extraction kit (Watson Biomedical Inc., Shanghai, China) and sequenced on an Applied Biosystem ABI 377 Sequencer using the Bigdye $^{\text {TM }}$. Terminator Cycle Sequencing kit (P. E. Biosystems Inc., Foster City, CA, USA). Both strands were sequenced using L15438, H16138, and an inner primer L15863 (5'-CCATCCTCCGTGAAACCAACA-3').

\section{Data analyses}

Sequences were aligned with DNASTAR software (DNASTAR Inc., Madison, WI, USA). All haplotypes, polymorphic sites and parsimony informative sites were identified with Mega 2.0 (Kumar et al. 2001). Published sequences and the haplotypes of European rabbits were used for comparison (Table 2). Relationships among the haplotypes were inferred in two ways. First, P-distances (proportional distance, proportion of nucleotide sites at which the two sequences are compared are different) between the sequences were used to construct a Neighbour-joining (NJ) tree with Mega 2.0 (Kumar et al. 2001). Bootstrap analyses (with 2000 replications) were used to assess the confidence in branching order. Some published sequences were excluded because they were shorter than our sequences. Secondly, a reduced median-joining network profile of the individuals was constructed according to Bandelt et al. (1995).

\section{Results}

\section{Sequence variation}

In the 104 individuals representing 20 breeds sequenced here (Table 1), 19 polymorphic sites were identified, with 18 transitions and one insertion/deletion. Eight haplotypes (A1, A2, A3, A4, A5, A6, A7 and A8) were resolved. Among the eight haplotypes, A1 was the most common and occurred in 89 individuals (Table 3). All other Chinese rabbits and the recently derived breeds except four individuals from strains $\mathrm{O}$ and $\mathrm{Y}$ shared haplotype A1. This
Table 1 Samples collected from 20 rabbit breeds in China.

\begin{tabular}{llll}
\hline Breed/strain (Abbreviation) & Locality & No. of samples & Origin \\
\hline Qixing rabbit (A) & Chengdu, Sichuan & 6 & Derived \\
Haerbin white rabbit (H) & Chengdu, Sichuan & 5 & Derived \\
Zhenhai thick-hair Angora rabbit (O) & Ningbo, Zhejiang & 5 & Derived \\
Big-ear brown rabbit (S) & Baoding, Hebei & 5 & Derived \\
Yufeng brown rabbit (Y) & Puyang, Henan & 5 & Derived \\
Belgium (B) & Chengdu, Sichuan & 5 & Introduced \\
Californian (C) & Chengdu, Sichuan & 4 & Introduced \\
Dwarf rabbit (D) & Chengdu, Sichuan & 4 & Introduced \\
ELCO (E) & Nanjing, Jiangsu & 5 & Introduced \\
Germany great line of ZIKA rabbit (G) & Chengdu, Sichuan & 5 & Introduced \\
New Zealand rabbit (J) & Jiangsu Nanjing & 5 & Introduced \\
Rex from United States (M) & Jiangsu Nanjing & 5 & Introduced \\
Germany New Zealand of Zika rabbit (N) & Chengdu, Sichuan & 4 & Introduced \\
Japanese white rabbit (R) & Kunming, Yunnan & 3 & Introduced \\
Rex from Germany (T) & Chengdu, Sichuan & 5 & Introduced \\
Angora rabbit from Germany (W) & Chengdu, Sichuan & 3 & Introduced \\
Germany white of Zika rabbit (Z) & Sichuan Chengdu & 5 & Introduced \\
Fujian brown rabbit (F) & Fuzhou, Fujian & 9 & Chinese \\
Taihang Moutain rabbit (P) & Baoding, Hebei & 6 & Chinese \\
Sichuan white rabbit (X) & Chengdu, Sichuan & 10 & Chinese \\
\hline
\end{tabular}


Table 2 Sequence data used in the study.

\begin{tabular}{|c|c|c|c|c|}
\hline \multirow[b]{2}{*}{ Accession No. } & \multicolumn{2}{|c|}{ Haplotype } & \multirow[b]{2}{*}{ Rabbit } & \multirow[b]{2}{*}{ References } \\
\hline & Original & This study & & \\
\hline AF003189 & Aus-1 & $\mathrm{A} 1$ & European rabbit in Australia & Zenger et al. (unpublished data) \\
\hline AF003190 & Aus-2 & A6 & European rabbit in Australia & Zenger et al. (unpublished data) \\
\hline AF003191 & Aus-3 & & European rabbit in Australia & Zenger et al. (unpublished data) \\
\hline AF003192 & Aus-4 & $\mathrm{A} 2$ & European rabbit in Australia & Zenger et al. (unpublished data) \\
\hline AF003193 & Aus-5 & & European rabbit in Australia & Zenger et al. (unpublished data) \\
\hline AF003194 & Aus- 6 & A3 & European rabbit in Australia & Zenger et al. (unpublished data) \\
\hline AF003195 & Aus-7 & & European rabbit in Australia & Zenger et al. (unpublished data) \\
\hline AJ293831 & B01Zsf & & Fauve de Bourgogne & Bolet et al. (2000) \\
\hline AJ293832 & B01Zsh & & Belgian hare & Bolet et al. (2000) \\
\hline AJ293833 & B01Zsg & & Fauve de Bourgogne & Bolet et al. (2000) \\
\hline AJ293834 & B01Zsj & & Argente de Champagne & Bolet et al. (2000) \\
\hline AJ293835 & B01Zsk & & English & Bolet et al. (2000) \\
\hline AJ293836 & B01Zsl & & Flemish giant & Bolet et al. (2000) \\
\hline AJ293837 & BZs14 & & Fauve de Bourgogne & Bolet et al. (2000) \\
\hline AJ293838 & BZs15 & & Hungarian Giant & Bolet et al. (2000) \\
\hline AJ293839 & BZs16 & & French Lop & Bolet et al. (2000) \\
\hline AJ293840 & BZs17 & & French Lop & Bolet et al. (2000) \\
\hline AJ293841 & BZs19 & & French Lop & Bolet et al. (2000) \\
\hline AJ293842 & $\mathrm{BZs} 20$ & A6 & Chinchilla & Bolet et al. (2000) \\
\hline AJ293843 & BZs21 & & Vienna White & Bolet et al. (2000) \\
\hline AJ293844 & Bzs22 & & Flemish Giant & Bolet et al. (2000) \\
\hline NC-001913 & & & & Gissi et al. (1998) \\
\hline U62924 & $A$ & A3 & wild rabbit in Australia & Fuller et al. (1997) \\
\hline U62925 & $\mathrm{B}$ & $\mathrm{A} 1$ & wild rabbit in Australia & Fuller et al. (1997) \\
\hline U62926 & $\mathrm{C}$ & A6 & wild rabbit in Australia & Fuller et al. (1997) \\
\hline U62927 & $\mathrm{D}$ & $\mathrm{A} 2$ & wild rabbit in Australia & Fuller et al. (1997) \\
\hline X54172 & & & & Mignotte et al. (1990) \\
\hline Z83340 & A5Zsa & & wild rabbit on Iberian Peninsula & van der loo et al. (1997) \\
\hline Z83341 & A2Zsa & & wild rabbit on Iberian Peninsula & van der loo et al. (1997) \\
\hline Z83342 & A10Zsa & & wild rabbit on Iberian Peninsula & van der loo et al. (1997) \\
\hline Z83343 & A1Zsa & & wild rabbit on Iberian Peninsula & van der loo et al. (1997) \\
\hline Z83344 & A9Zsa & & wild rabbit on Iberian Peninsula & van der loo et al. (1997) \\
\hline Z83346 & B10Zsa & & rabbit in Spain & van der loo et al. (1997) \\
\hline Z83349 & B6Zsa & & rabbit in Spain & van der loo et al. (1997) \\
\hline Z83350 & B4Zsa & & rabbit in Spain & van der loo et al. (1997) \\
\hline Z83351 & B3Zsc & & rabbit in Spain & van der loo et al. (1997) \\
\hline Z83354 & Bzs1 & & rabbit in Spain & van der loo et al. (1997) \\
\hline Z83364 & B2Zsa & & rabbit in Spain & van der loo et al. (1997) \\
\hline Z83365 & B3zsa & $\mathrm{A} 2$ & rabbit in Spain & van der loo et al. (1997) \\
\hline Z83366 & $\mathrm{B} 2 \mathrm{zsC}$ & $\mathrm{A} 3$ & rabbit in Spain & van der loo et al. (1997) \\
\hline Z83367 & B1zsb & A1 & rabbit in Spain & van der loo et al. (1997) \\
\hline
\end{tabular}

haplotype was also observed in all introduced animals except strain G.

Alignment of these eight haplotypes and the reported sequences of European rabbit (Table 2) revealed 37 unique haplotypes. The current study resolved four new haplotypes (A4, A5, A7 and A8), and the other four haplotypes (A1, A3, A6, A2) were identical to previously published haplotypes (Table 2).

\section{Phylogenetic analysis}

The unrooted NJ tree of wild and domesticated rabbit mtDNA sequences is shown in Fig. 1. Two lineages (A and B) were clearly discerned, with lineage A composed of wild rabbits and lineage $B$ including both domestic and wild animals. In lineage B, two clusters were discerned. Six haplotypes in the present study (A1, A4, A5, A7, A8 and A3) were scattered in the first cluster and the remaining two haplotypes (A2 and A6) belonged to the second cluster. 
Table 3 Frequencies of eight mitochondrial DNA haplotypes in 20 rabbit breeds in China.

\begin{tabular}{|c|c|c|c|c|c|c|c|c|}
\hline \multirow[b]{2}{*}{ Breed/strain ${ }^{1}$} & \multicolumn{8}{|l|}{ Haplotype } \\
\hline & $\mathrm{A} 1$ & A2 & A3 & A4 & A5 & A6 & A7 & A8 \\
\hline A & $6(1.00)$ & - & - & - & - & - & - & - \\
\hline B & $5(1.00)$ & - & - & - & - & - & - & - \\
\hline C & $4(1.00)$ & - & - & - & - & - & - & - \\
\hline D & $4(1.00)$ & - & - & - & - & - & - & - \\
\hline $\mathrm{E}$ & $5(1.00)$ & - & - & - & - & - & - & - \\
\hline $\mathrm{F}$ & $9(1.00)$ & - & - & - & - & - & - & - \\
\hline G & - & $5(1.00)$ & - & - & - & - & - & - \\
\hline $\mathrm{H}$ & $5(1.00)$ & - & - & - & - & - & - & - \\
\hline J & $4(0.80)$ & $1(0.20)$ & - & - & - & - & - & - \\
\hline$M$ & $4(0.80)$ & - & - & - & - & - & - & $1(0.20)$ \\
\hline$N$ & $4(1.00)$ & - & - & - & - & - & - & - \\
\hline O & $3(0.60)$ & - & $2(0.40)$ & - & - & - & - & - \\
\hline$P$ & $6(1.00)$ & - & - & - & - & - & - & - \\
\hline$R$ & $2(0.67)$ & - & - & $1(0.33)$ & - & - & - & - \\
\hline$S$ & $5(1.00)$ & - & - & - & - & - & - & - \\
\hline $\mathrm{T}$ & $4(0.80)$ & $1(0.20)$ & - & - & - & - & - & - \\
\hline W & $3(1.00)$ & - & - & - & - & - & - & - \\
\hline$x$ & $10(1.00)$ & - & - & - & - & - & - & - \\
\hline Y & $3(0.60)$ & - & - & - & $1(0.20)$ & $1(0.20)$ & - & - \\
\hline Z & $3(0.60)$ & - & - & - & - & - & $1(0.20)$ & $1(0.20)$ \\
\hline total & $89(0.85)$ & $7(0.07)$ & $2(0.02)$ & $1(0.01)$ & $1(0.01)$ & $1(0.01)$ & $1(0.01)$ & $2(0.02)$ \\
\hline
\end{tabular}

${ }^{1}$ Abbreviations for breeds are given in Table 1 . Numbers of individuals that shared a haplotype are given outside the brackets; frequencies of the haplotypes are bracketed.

The sequences in this study have been submitted to GenBank and the accession numbers are AF534080-AF534108.

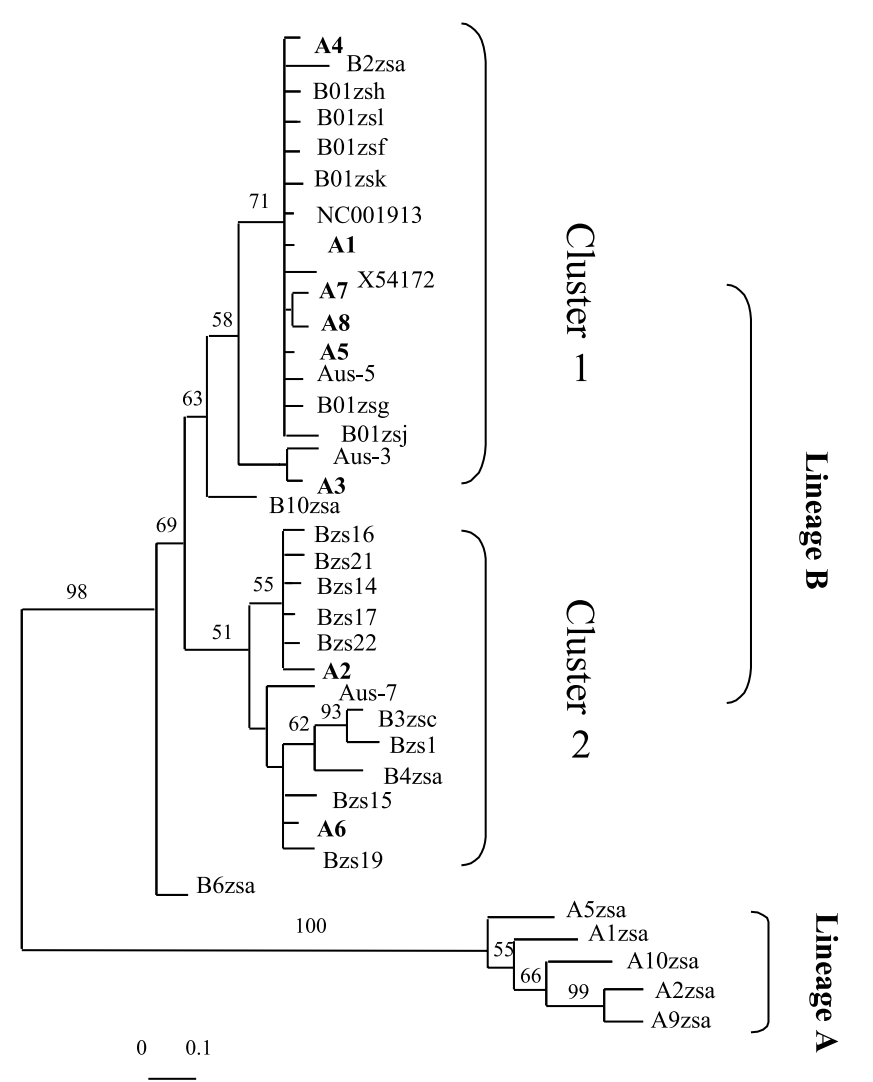

Figure 1 Unrooted Neighbour-joining (NJ) tree for 37 mtDNA sequences of rabbits using uncorrected P-distances. Numbers on the branches are percentage of bootstrap values from 2000 replications. The haplotypes identified in current study are in bold. 


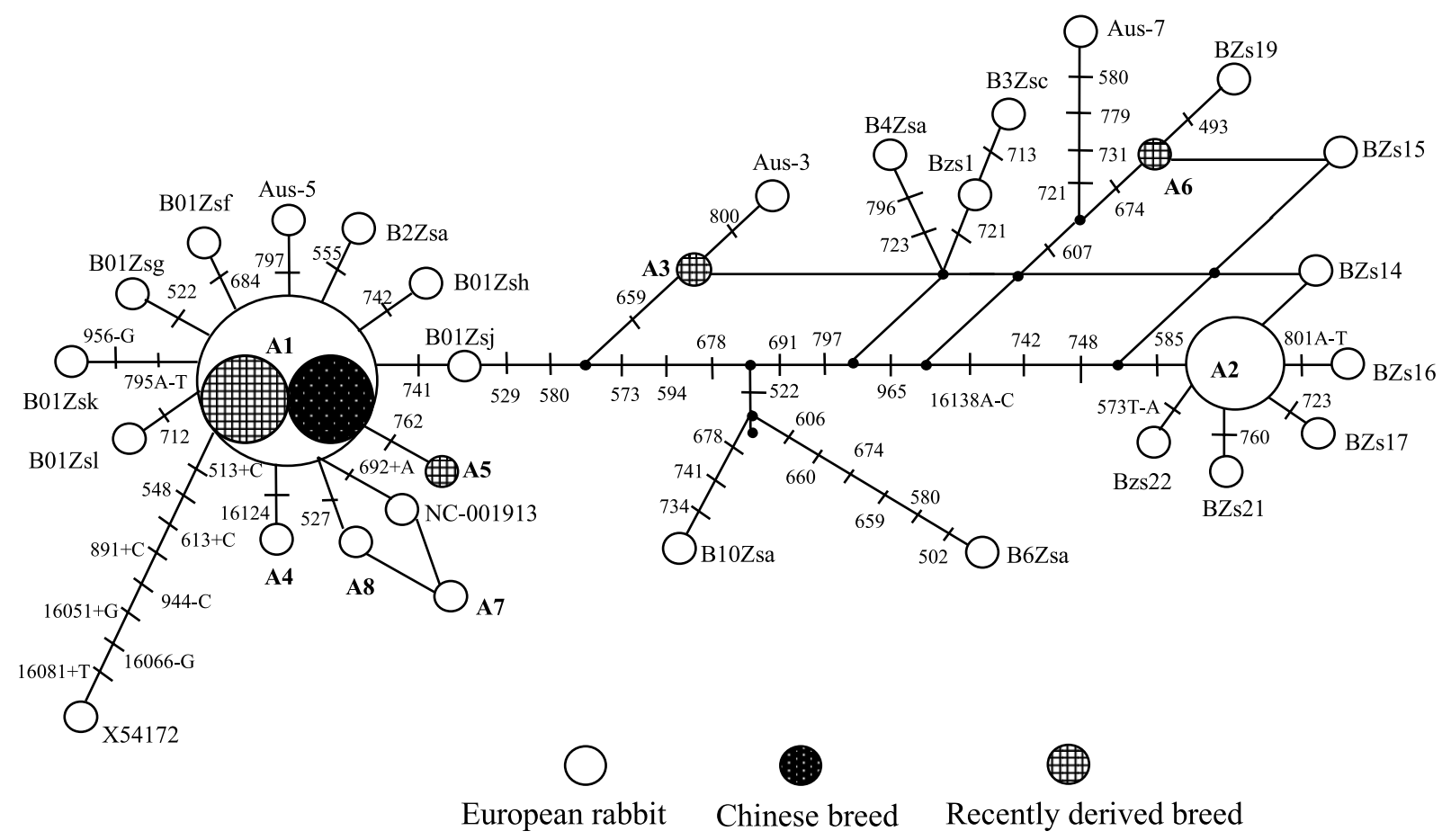

Figure 2 Network profiles of $32 \mathrm{mtDNA}$ haplotypes. Each circle represents one haplotype with a size proportional to the number of individuals. The black points represent hypothetical sequences that were not observed. Branches between the haplotypes represent substitutions numbered according to Gissi et al. (1998). Site numbers below 16000 are marked only the last three digits. The transversion and insertion/deletion are labelled. Recurrent mutations are underlined, designating putative distinct mutation events at the same position. The haplotypes identified in current study are in bold.

In the network connecting 32 haplotypes of lineage $B$ (Fig. 2), the highly represented haplotypes A1 and A2 were centred with the other sequences rooted through these haplotypes. Most sequences fell within only a few substitutions of the central nodes. Haplotypes A4, A5 and A8 differed in only one site from haplotype A1. There were two and four substitutions between A7 and A1, and A3 and A1, respectively. In the present study, nine introduced strains (B, C, D, E, M, N, R, W and Z), all Chinese breeds (F, P and X), and four recently derived breeds (A, H, O and S) were scattered in the first cluster. The second cluster was centred on haplotype A2. All individuals from strain $G$ belonged to this cluster. Two introduced strains ( $\mathrm{J}$ and $\mathrm{T}$ ) and one recently derived breed $(\mathrm{Y})$ were intermingled in the two clusters.

\section{Discussion}

\section{Differentiation between domesticated rabbit strains}

In the present study, 19 strains shared haplotype A1 and three strains shared haplotype A2, respectively (Table 3). Thus mtDNA differences were not distinct among the 20 strains, although their morphological differences were large. The results suggested that genetic diversity was low both in the Chinese rabbits and the introduced strains. This low genetic diversity might be due to: (1) founder effects during domestication because of the limited introduction of genes from the wild population, and when imported from Europe (see Discussion below), (2) during cultivation of a strain, homogeneity mating occurred, resulting in genetic monotony, (3) in commercial animal production, selection pressure was high, which drastically decreased the effective number $\left(N_{\mathrm{e}}\right)$ and the inherent variability of the strain, and (4) crossbreeding decreased genetic diversity in some strains. As a result, the maternally inherited mtDNA haplotypes of breeds from which only bucks were used, will be lost.

\section{Origin of rabbits in China}

In general, it is thought that there are two maternal lineages in the European rabbit based on data of mtDNA and immunoglobulin genes (Biju-Duval et al. 1991; Monnerot et al. 1994; van der Loo et al. 1997; Branco et al. 2000). All domestic rabbits belong to the second lineage (Monnerot et al. 1996).

The origin of Chinese rabbits is controversial. Sequence comparisons and phylogenetic analyses indicated only one mtDNA haplotype (A1) was represented in Chinese rabbits (Table 3). Some European breeds were imported into China and they might introgress into Chinese animals, resulting in a decrease in genetic diversity of Chinese rabbits. However, 
the number of imported rabbits was very small and the effects of European animals were limited. Thus, the founders of Chinese rabbit probably originated from a population that carried haplotype A1. Haplotype A1 was commonly found (about 70\%) in many European domestic breeds, such as Fauve de Bourgogne, Argenté de Champagne, and Flemish giant (Bolet et al. 2000), and in wild populations from France (M Monnerot, personal communication) as well as Australia (K Zenger, unpublished data; Fuller et al. 1997). Chinese breeds, some of the recently derived breeds in China, and some European rabbits were intermingled in the cluster centred with haplotype A1 (Fig. 2). Therefore, a more plausible scenario is that the so-called Chinese rabbits were also introduced from European rabbits.

\section{Acknowledgements}

We thank Yong-Gang Yao for helpful comments on an earlier version of the manuscript, Gou Shi-Kang for technical assistance, and Dr Monnerot Monique for good suggestions. We are also grateful to Xie Xiao-Hong (Sichuan Academy of Animal Sciences), Liu Han-Zhong (Grass Institute of Sichuan Province), Lou Zhi-Rong (Animal Bureau of Jiangsu Province), Professor Gu Zi-Lin (Hebei Agricultural University) and Zhan Jian-Liang (Zhenhai Wool Rabbitry) for assistance in collecting samples. This work was supported by the State Key Basic Research and Development Plan (G20000161), NSFC and Yunnan Province.

\section{References}

Ban, Z.H., Liu, R.Y., Xiao, C.N. et al. (1996) Feasibility study on calculating Heterosis by genetic structure of strains. Chinese Journal of Rabbit Farming 1, 15-21 (in Chinese).

Bandelt, H.J., Forster, P., Sykes, B.C. \& Richands, M.B. (1995) Mitochondrial portraits of human populations using median networks. Genetics 141, 743-53.

Biju-Duval, C., Ennafaa, H., Dennebouy, N. et al. (1991) Mitochondrial DNA evolution in lagomorphs: origin of systematic heteroplasmy and organization of diversity in European rabbits. Journal of Molecular Evolution 33, 92-102.

Bolet, G., Brun, J.M., Monnerot, M. et al. (2000) Evaluation and conservation of European rabbit (Oryctolagus cuniculus) genetic resources. First results and inferences. 7 th World Rabbit Congress A, 281-316.
Branco, M., Ferrand, N. \& Monnerot, M. (2000) Phylogeography of the European rabbit (Oryctolagus cuniculus) in the Iberian Peninsula inferred from RFLP analysis of the cytochrome $b$ gene. Heredity 85, 307-17.

Chen, Y.W. (1984) Discussion on the origin of the domestic rabbit in China. Chinese Journal of Rabbit Farming 2, 37-43 (in Chinese).

Chen, Y.W. \& Wang, Y.H. (1991) The history of Chinese rabbit farming. Chinese Journal of Rabbit Farming 5, 5-13 (in Chinese).

Ennafaa, H., Monnerot, M., Gaaied, A.E. \& Mounolou, J.C. (1987) Rabbit mitochondrial DNA: preliminary composition between some domestic and wild animals. Genetics, Selection, Evolution 3, 279-88.

Fuller, S.J., Wilson, J.C. \& Mather, P.B. (1997) Patterns of differentiation among wild rabbit populations Oryctolagus cuniculus L. arid and semiarid ecosystems of north-eastern Australia. Molecular Ecology 2, 145-53.

Gissi, C., Gullberg, A. \& Arnason, U. (1998) The complete mitochondrial DNA sequence of the rabbit, Oryctolagus cuniculus. Genomics 2, 161-9.

Hardy, C., Callou, C., Vigne, J.D., Casane, D., Dennebouy, N., Mounolou, J.C. \& Monnerot, M. (1995) Rabbit mitochondrial DNA diversity from prehistoric to modern times. Journal of Molecular Evolution 3, 227-37.

Kumar, S., Tamura, K., Jakobsen, I.B. \& Nei, M. (2001) MEGA2: molecular evolutionary genetics analysis software. Bioinformatics 12, 1244-5.

Luo, Z.X. (1988) The Chinese Hare. China Forestry Publishing House, Beijing. pp. 18-22 (in Chinese).

Mignotte, F., Gueride, M., Champagne, A.M. \& Mounolou, J.C. (1990) Direct repeats in the non-coding region of rabbit mitochondrial DNA. Involvement in the generation of intra- and inter-individual heterogeneity. European Journal of Biochemistry 2, 561-71.

Monnerot, M., Loreille, O., Mougel, F., Vachot, A.M., Dennebouy, N., Callou, C., Vigne, J.D. \& Mounolou, J.C. (1996) The European rabbit: wild population evolution and domestication. 6th World Rabbit Congress, 331-4.

Monnerot, M., Vigne, J.D., Biju-Duval, C., Casane, D., Callou, C., Hardy, C., Mougel, F., Soriguer, R., Dennebouy, N. \& Mounolou, J.C. (1994) Rabbit and man: genetic and historic approach. Genetics, Selection, Evolution 1, 167-82.

van der Loo, W., Mougel, F., Sanchez, M.S., Bouton, C., Castien, E., Soriguer, R., Hamers, R. \& Monnerot, M. (1997) Evolutionnary patterns at the antibody constant region in rabbit (Oryctolagus cuniculus): characterisation of endemic b-locus allotypes and their frequency correlation with major mitochondrial gene types in Spain. Gibier Faune Sauvage 14, 427-49. 